\begin{tabular}{|c|c|}
\hline Title & A critical condition for the formation of a coarse columnar gamma grain structure in a peritectic solidified carbon steel \\
\hline Author(s) & Ohno, Munekazu; Maruyama, Masato; Matsuura, Kiyotaka \\
\hline Citation & $\begin{array}{l}\text { A cta materialia, 61(19), } 7334.7341 \\
\text { https://doi.org/10.1016j.actamat.2013.08.039 }\end{array}$ \\
\hline Issue Date & $2013-11$ \\
\hline Doc URL & http://hdl.handle.net/2115/54711 \\
\hline Tyре & article (author version) \\
\hline File Information & 20140hno_HUSCAP_.pdf \\
\hline
\end{tabular}

Instructions for use 


\title{
Critical condition for formation of coarse columnar $\gamma$ grain structure in a peritectic solidified carbon steel
}

\author{
Munekazu Ohno ${ }^{a^{*}}$, Masato Maruyama ${ }^{\text {b, Kiyotaka Matsuura }}{ }^{\text {a }}$ \\ ${ }^{a}$ Division of Materials Science and Engineering, Faculty of Engineering, Hokkaido University, Kita \\ 13 Nishi 8, Kita-ku, Sapporo, Hokkaido 060-8628, Japan \\ ${ }^{b}$ Graduate School of Engineering, Hokkaido University \\ * Corresponding author. Address: Division of Materials Science and Engineering, Faculty of \\ Engineering, Hokkaido University, Kita 13 Nishi 8, Kita-ku, Sapporo, Hokkaido 060-8628, Japan. \\ Tel/Fax: +81 11706 6344. E-mail address: mohno@eng.hokudai.ac.jp
}

\begin{abstract}
The formation of coarse columnar austenite grain (CCG) structure is one of the serious problems in continuous casting processes of peritectic solidified carbon steels. In this study, a guiding principle for avoidance of the CCG formation is developed. The critical condition of the CCG formation recently put forward based on phase-field simulations, which is given by a balance between the cooling condition and the growth rate of the CCG, is first reexamined and modified by considering effect of liquid phase during the CCG formation. Then, the validity of this critical condition is investigated by three different casting experiments combined with heat conduction analyses. From comparison between the cooling conditions and the resulting microstructural changes, the validity of the critical condition is successfully demonstrated.
\end{abstract}

Keywords: Casting; As-cast austenite grain; Solidification microstructure; Peritectic reaction 


\section{Introduction}

As-cast $\gamma$-austenite grain structures in continuously cast slabs of peritectic solidified carbon steels consist of coarse columnar grains (CCGs) in the vicinity of the slab surface [1, 2]. The formation of CCGs deteriorates the hot ductility of the slabs and it causes the occurrence of surface cracking [3-6]. It was reported that the surface crack propagates along the grain boundary of CCGs [3] and the depth of the surface crack is affected by the major axis diameter of CCGs. Hence, it is necessary to precisely control the as-cast $\gamma$ grain structure so as to produce equiaxed $\gamma$ grains (EGs) instead of CCGs. However, such microstructure control has been a longstanding issue remaining unresolved in the field of casting of steels, because there has been no reliable guiding principle established for the avoidance of the formation of CCGs.

The mechanism of formation of CCGs in hyperperitectic carbon steels was investigated in our recent works [7, 8] using rapid unidirectional solidification equipment which realizes the cooling conditions similar to those near the slab surface in the practical continuous casting. The schematic illustration of the microstructure during the formation process of CCGs is shown in Fig. 1(a). It was found that during the formation process of CCGs, fine columnar $\gamma$ grains (FCGs) always exist ahead of the CCGs growing along the temperature gradient ( $y$ direction). The change from the FCG to CCG structure is discontinuous and the boundary between these two regions can be recognized very clearly [7]. This boundary is called the FCG/CCG region boundary (FCRB) in this paper. The temperature at the FCRB is always close to a temperature for completion of transformation to $\gamma$ single phase, $T_{\gamma}$, during the solidification. Hence, the FCG region corresponds to the liquid $+\gamma$ two-phase field, while the CCG region is in $\gamma$ single-phase field. The short axis diameter of FCGs is comparable to the primary dendrite spacing or distance between the dendrite trunks of the $\delta$ phase, because the interdendritic liquid phase in the FCG region inhibits the growth of FCGs along the short axis direction. As the cooling proceeds, the position of $T_{\gamma}$ moves away from the mold side ( $y$ direction) and the liquid phase accordingly disappears in this region. The FCRB then moves away from the mold side, which corresponds to the growth of CCGs and the shrinkage of FCGs. This process is characterized as the discontinuous grain growth [7]. It is noted that this process was observed in hyperperitectic carbon steels where the peritectic reaction and transformation take place during cooling [7, 8]. When the carbon concentration becomes high, for example, when it is higher than 0.43 mass $\%$ $\mathrm{C}$ in the rapid unidirectional solidification experiment, the as-cast $\gamma$ grain structure is entirely determined by $\gamma$ solidification process because the preferential growth of $\gamma$ 
dendrite instead of $\delta$ dendrite takes place [8]. Hence, the discontinuous growth does not arise in this case. The main concern in this paper is the former case, viz., the hyperperitectic carbon steels where the peritectic reaction and transformation takes place because these steels are more prone to exhibiting coarse $\gamma$ grain structures.

As described above, the formation of CCGs is ascribable to the occurrence of discontinuous grain growth from the FCG structure. It is therefore necessary for the prevention of the CCG formation to elucidate the condition for the discontinuous grain growth to take place. In our recent study [9], the formation process of CCGs from FCGs was examined by means of phase-field simulations for the grain growth. It was shown that although the continuous grain growth yields the EG structure, the discontinuous grain growth results in the CCG structure, which is in agreement with the experimental finding [7, 8]. Furthermore, we derived the critical condition for the discontinuous growth to start and continue, which is given by [9],

$$
V_{T}=M\left(T_{\gamma}\right) \frac{1}{\xi d_{F C G}}
$$

where $V_{\mathrm{T}}$ is the migration velocity of $T_{\gamma}$ position called the thermal velocity, $d_{\mathrm{FCG}}$ is the short axis diameter of FCG and $\xi$ is a parameter determined by the local shape of FCRB. $M\left(T_{\gamma}\right)$ is a kinetic constant for the grain growth at $T_{\gamma}$, which is closely related to the grain boundary mobility. When the temperature gradient is extremely high, correction due to the temperature gradient is required for $M\left(T_{\gamma}\right)$ [9] but this correction is not necessary in usual casting conditions and also in the present case. This equation will serve as the guiding principle for the prevention of the CCG formation. One can design the cooling condition and/or chemical compositions of steels based on Eq. (1) so as to obtain the EG structure instead of the CCG structure.

It should be pointed out that the critical condition given by Eq. (1) was verified only by the phase-field simulations for the grain growth without considering the full details of the solidification processes. For simplicity, an effect of the liquid phase on the motion of FCRB was omitted in the derivation of Eq. (1), while the liquid phase always exists near the FCRB during the formation process of CCGs [7]. In reality, therefore, the effect of the liquid should not be negligible and, in this respect, the critical condition needs to be reexamined. More importantly, the validity of the critical condition, Eq.(1), has not been experimentally demonstrated and the experimental verification is indispensable for practical applications of Eq. (1). These issues are tackled in this study. First the critical condition is modified by taking the effect of liquid phase on the motion of FCRB into account. It simply results in a modification in the value of $\xi$ in Eq. (1). In order to test the validity of Eq. (1) with the new value of $\xi$, then, we carry out three 
types of casting experiments and analyze the resulting as-cast $\gamma$ grain structures. The transition of structure from CCGs to EGs is compared to the behavior of $V_{\mathrm{T}}$ obtained from heat conduction analysis for each casting process. One will see that the validity of Eq. (1) is successfully substantiated by means of the present casting experiments.

\section{Critical condition of CCG formation}

In the previous work [9], the critical condition given by Eq. (1) was derived and analyzed based on the phase-field simulations for the CCG formation. For the sake of convenience, an effect of the liquid phase on the motion of FCRB was omitted in the simulations and the condition of Eq. (1) was accordingly derived without considering such an effect. In reality, however, this effect may not be negligible as discussed later. In this study, we take this effect into consideration and modify the critical condition. For the clear exposition, our discussion starts with the derivation of Eq. (1) without the effect of liquid phase on the motion of FCRB.

According to the theory developed by Humphreys [10], the discontinuous grain growth takes place when the grain boundary mobility of a (coarse) grain is higher than that of the other (fine) grains. During the formation process of CCGs, the grain boundary mobility of FCGs should be extremely small as long as there is the liquid phase exerting the strong pinning force on the growth of FCGs. As the cooling proceeds, the liquid phase disappears and the pinning effect accordingly vanishes in the FCG region. Then, the mobility of FCGs becomes higher than that of CCGs, because of the higher temperature in the FCG region. Hence, when $V_{\mathrm{T}}$ is lower than the velocity of FCRB moving away from the mold $V_{\mathrm{CCG}}$, the discontinuous grain growth occurs, because the growth of FCGs does not take place ahead of the growing CCGs. On the other hand, when $V_{\mathrm{T}}>V_{\mathrm{CCG}}$, the FCGs can grow before the migration of FCRB, thus preventing the CCGs from growing. Therefore, it should be reasonable to postulate that the critical condition for the CCG formation is given by $V_{T}=V_{\mathrm{CCG}}$.

The migration velocity of FCRB, $V_{C C G}$, corresponds to the velocity of shrinkage of FCGs. Based on the theory of the grain growth developed by Hillert [11], the relation $V_{\text {CCG }}=M\left(T_{\gamma}\right) / r$ can be derived [9] where $r$ is the curvature radius of the FCRB. Shown in Fig. 1(b) is the schematic illustration of three-dimensional shape of the FCRB where the cross-sectional shape of the FCGs on $x-z$ plane is assumed as a regular hexagon. The directions of the principal curvatures of the FCRB correspond to $x$ and $z$ directions and the radii of the curvatures in these directions are denoted by $r_{1}$ and $r_{2}$. Using these radii, $r$ is given by $1 / r=\left(1 / r_{1}+1 / r_{2}\right) / 2$. When the short and long diagonals of the hexagon are specified by $d_{1}$ and $d_{2}$, the radii of the curvatures can be 
expressed as $r_{1}=\xi_{1} d_{1}$ and $\xi_{2} d_{2}$ with constants $\xi_{1}$ and $\xi_{2}$. These radii are also expressed as $r_{1}=2 \xi_{1} a$ and $r_{2}=\sqrt{3} \xi_{2} a$ with the side length of the hexagon $a$. When $a$ is given by $a=q d_{\mathrm{FCG}}$ with a constant $q, V_{\mathrm{CCG}}$ is rewritten as

$$
V_{\mathrm{CCG}}=M\left(T_{\gamma}\right) \frac{1}{\xi d_{F C G}},
$$

where

$$
\frac{1}{\xi}=\left(\frac{1}{2 \xi_{1}}+\frac{1}{\sqrt{3} \xi_{2}}\right) \frac{1}{2 q} .
$$

The substitution of Eq. (2) into the relation $V_{T}=V_{\text {CCG }}$ yields the critical condition of the CCG formation given by Eq. (1).

In our previous study [9], $\xi_{1}$ and $\xi_{2}$ were determined from the local shape of FCRB based on the Young's law. The schematic illustration of the FCRB on $x-y$ plane (dashed rectangle in Fig. 1(b)) is shown in Fig. 1(c). Using $\theta_{2}$ defined in Fig. 1(c), $\xi_{2}$ is given by $\xi_{2}=\left(1+\tan ^{2} \theta_{2}\right)^{1 / 2} /\left(2 \tan \theta_{2}\right)=1 /\left(2 \sin \theta_{2}\right)$. If the grain boundary energy is isotropic, the contact angle at the triple junction is $120^{\circ}$ and hence $\theta_{2}=30^{\circ}$ and $\xi_{2}=1$. When the same argument is applied to $\xi_{1}$, it can be estimated to be $\xi_{1} \cong 0.87$. In the previous numerical study [9], $q$ was approximated by $q \cong \pi^{1 / 2} / 3^{1.25}$ based on a consideration of geometry of grains and, finally, $\xi$ was calculated to be $\xi=0.78$. It was shown in the previous work [9] that the critical condition given by Eq. (1) is completely consistent with the results of the phase-field simulations for the CCG formation.

In the discussion about $V_{\mathrm{CCG}}$ described above, the existence of liquid phase is ignored. However, when $V_{\mathrm{T}}$ is close to $V_{\mathrm{CCG}}$, the liquid phase exists near the FCRB and the local shape of FCRB is not determined by the local equilibrium at the triple junction of the grain boundaries. Figure 2(a) schematically represents the FCRB on $x$-y plane when the liquid phase exists. The energetically unfavorable triple junction should be covered by the liquid phase and the FCRB thereby contacts with the liquid phase directly. In order to discuss the local shape of FCRB precisely, it is necessary to know relative magnitudes of the grain boundary energy $\sigma_{\mathrm{g}}$ and the $\gamma$-liquid interfacial energy $\sigma_{\gamma / L}$. Although it is generally considered that the relation $\sigma_{\mathrm{g}}>\sigma_{\gamma / L}$ is satisfied $[12,13]$, these exact values are not known for the hyperperitectic steels of our concern. For simplicity, the present discussion is given for the case of $\sigma_{\mathrm{g}}<2 \sigma_{\gamma / L}$ where the infiltration of liquid phase into the grain boundary does not arise. In addition, $\sigma_{\mathrm{g}}$ and $\sigma_{\gamma / L}$ are assumed as isotropic. As shown in Fig. 2(b), there exists the triple junction consisting of the FCRB and two $\gamma$-liquid interfaces. In this case, the contact angle between the FCRB and the $\gamma$-liquid interface is denoted by $\theta_{\Gamma}$. When the liquid phase disappears upward at the velocity $V_{\mathrm{T}}$, the FCRB can move at the same velocity as long as the curvature radius 
of FCRB is sufficiently small. On the other hand, when the curvature radius of FCRB is not small, the FCRB seems not to be able to move at $V_{\mathrm{T}}$. In this case, however, the FCRB should be elongated and curved in accordance with the migration of the triple junction as shown in Fig. 2(c). Consequently, the curvature radius of the FCRB decreases and $V_{\mathrm{CCG}}$ thereby increases. In the extreme case, the triple junction should be located at the end edge of the disappearing liquid phase and the curvature radius of the FCRB is as small as the radius of circumscribed circle of the regular hexagonal FCG, viz., $r=a$. In this case, $\xi$ is estimated to be $\xi=0.45$. This gives the maximum velocity of $V_{\mathrm{CCG}}$ which is roughly twice as high as that for $\xi=0.78$. When $V_{\mathrm{T}}$ becomes higher than this maximum velocity, the FCRB leaves the liquid phase and touches the

neighboring FCRB, forming the triple junction of the grain boundaries. Then, the discontinuous grain growth ceases because the grain growth of FCGs takes place ahead of CCGs.

As discussed above, the motion of FCRB should be accelerated by the disappearance of liquid phase contacting with the FCRB. As reported elsewhere [14], the occurrence of this acceleration is actually confirmed by the phase-field simulations. Although some simplifications were introduced in the above-mentioned discussion and, in particular, very simplified shape of the disappearing liquid phase (last solidifying liquid) was considered, $\xi=0.45$ is expected to yield better accuracy of Eq. (1). In fact, this is the important correction to improve the quantitative accuracy of the critical condition given by Eq. (1) as demonstrated in Section 4.

\section{Experimental and numerical procedures}

In order to test the validity of Eq. (1) with $\xi=0.45$, three types of casting experiments were carried out by employing the different set of molds. Moreover, the behavior of $V_{\mathrm{T}}$ during the casting processes was examined by the heat conduction analysis. The details are explained below.

We focused on a hyperperitectic carbon steel, the chemical composition of which is $0.2 \% \mathrm{C}, 0.21 \% \mathrm{Si}, 0.81 \% \mathrm{Mn}, 0.02 \% \mathrm{P}, 0.004 \% \mathrm{~S}, 0.04 \mathrm{Al}$, and $0.0064 \% \mathrm{~N}$ (in mass\%). The sample of $250 \mathrm{~g}$ put in a cylindrical $\mathrm{MgO}$ crucible with an inner diameter of $28 \mathrm{~mm}$ and a depth of $90 \mathrm{~mm}$ was melted at $1570^{\circ} \mathrm{C}$ in a SiC furnace filled with $\mathrm{Ar}$ gas of five-nines purity. It was held at this temperature for $1 \mathrm{~h}$ and then the melted sample was cast into the mold held at room temperature before the casting. Figure 3(a) is the schematic illustration of the casting equipment employed in this study. It consists of a refractory brick stage $\left(62 \% \mathrm{SiO}_{2}-33 \% \mathrm{Al}_{2} \mathrm{O}_{3}\right)$ and three types of mold parts as specified by the mold 1, 2 and 3 . In this study, we carried out three types of the casting 
experiments. In the first experiment, all the mold parts (mold 1, 2 and 3) are composed of the steel molds with a thickness of $L=20 \mathrm{~mm}$ and this is called the metallic-metallic mold casting. In the second one, all the mold parts are the $\mathrm{Al}_{2} \mathrm{O}_{3}$ molds with $L=10 \mathrm{~mm}$ and it is called the ceramic-ceramic mold casting. In the third experiment, the mold 1 is the steel mold, while the mold 2 and 3 are the $\mathrm{Al}_{2} \mathrm{O}_{3}$ molds. This last one is called the metallic-ceramic mold casting. The casting with the different molds will yield the different cooling process and thus the different $\gamma$ grain structure.

In all the casting experiments, the shape of the cast sample was a rectangular prism with a height of $40 \mathrm{~mm}$, a width of $40 \mathrm{~mm}$ and a thickness of $20 \mathrm{~mm}$. The sample was sectioned horizontally at a height of $20 \mathrm{~mm}$. A rectangular area on the sectioned surface, extending from one mold side to the other mold side in the thickness direction, was selected for the microstructural observation. It is allowed to take into account only the one-dimensional heat flow in the thickness direction on this selected area [15]. This area was polished and etched with 3\% nital and Oberhoffer's solutions to observe the as-cast $\gamma$ grain and dendrite structures, respectively.

In addition to the metallographic investigation, the temperature measurements were carried out during the casting processes. The cooling curves at the different positions in the samples were preliminarily monitored by setting B-type thermocouples inside the samples at 1 and $6 \mathrm{~mm}$ away from the mold in the metallic-metallic mold casting, $1 \mathrm{~mm}$ away from the mold in the ceramic-ceramic mold casting and $1 \mathrm{~mm}$ away from the metallic mold and $4 \mathrm{~mm}$ away from the ceramic mold in the metallic-ceramic mold casting. These cooling curves at the different positions were utilized to obtain the heat-transfer coefficients for numerical simulations of the heat conduction process. The standard heat conduction equation including the effect of latent heat [16] was solved in a one-dimensional system consisting of the cast and molds based on the equivalent heat capacity method. The mesh size was set to $\Delta x=5 \times 10^{-6} \mathrm{~m}$ and the heat capacity and the latent heat were obtained from the CALPHAD method with PanIron database [17] and the other parameters were taken from Refs. [16, 18]. By means of the numerical simulations thus described, the behavior of $V_{\mathrm{T}}$ in each casting experiment was analyzed.

\section{Results and discussion}

\subsection{As-cast $\gamma$ grain and dendrite structures}

The as-cast $\gamma$ grain and dendrite structures in the same region of the sample cast in the ceramic-ceramic mold are shown in Figs. 3(b) and (c), respectively. The bottom and top parts of these figures corresponds to the mold sides. In Fig. 3(b), some grain boundaries are traced by thin lines for visual aid. The position where the structure 
changes from the CCG to EG is indicated by the thick line and, hereafter, this position is called the transition depth. The CCGs form near the mold side, while the EGs form in the center region. In Fig. 3(c), the dendrite structure consists of the columnar dendrites developing inward from the mold side and the equiaxed dendrite in the center region. The position of the transition between them is indicated by the thick line in Fig. 3(c). It is clearly observed that the CCG region does not exactly corresponds to the region of the columnar dendrite. For example, when the top-left part of these structures are considered, the CCGs stop growing inward at about $4 \mathrm{~mm}$ depth from the mold side (top side), whereas the columnar dendrites develop inward up to about $7 \mathrm{~mm}$ depth from the mold side.

The discrepancy between the $\gamma$ grain and dendrite structures is more evident in the sample cast in the metallic-ceramic mold as shown in Figs. 4 (a) and (b). The bottom and top sides of these figures correspond to the metallic and ceramic mold sides, respectively. In this sample, the CCGs develop from the metallic mold, while the only EGs form in the ceramic mold side. The transition depth is located near the center of the sample. On the other hand, the columnar dendrite develops from both sides in Fig. 4(b). Although the columnar dendrite region in the ceramic mold side is shorter than that in the metallic mold side, the columnar dendrite region extends to more than $3 \mathrm{~mm}$ depth from the ceramic mold. The magnified views of the structure near the ceramic mold are shown in Figs. 4(c) and (d). It is clearly seen that the long columnar dendrite develops in this region, while the $\gamma$ grains are equiaxed and the grain diameter is shorter than the dendrite length. Hence, it is understood that there is no correlation between the shapes of $\gamma$ grains and dendrites. This fact supports that the CCGs form by the discontinuous grain growth mechanism.

Although not shown here, the structures in the metallic-metallic mold casting are similar to those in the ceramic-ceramic casting. The transition depths are different in the samples cast in the different molds. The critical condition given by Eq. (1) is considered to be realized at the transition depth in each casting condition. These transition depths are compared with the calculated behavior of $V_{\mathrm{T}}$ below.

\subsection{Behavior of the thermal velocity}

Figure 5 represents the calculated results of thermal velocity $V_{\mathrm{T}}$ with respect to the distance from the mold. The results for the metallic-metallic mold (solid line with square plots) and ceramic-ceramic mold (solid line with circle plots) casting are shown in Fig. 5(a) where the only region of less than $y=10 \mathrm{~mm}$ is displayed because the behavior of $V_{\mathrm{T}}$ is symmetric with respect to $y=10 \mathrm{~mm}$. The behavior of $V_{\mathrm{T}}$ clearly 
depends on the type of molds. The values of $V_{\mathrm{T}}$ in the metallic-metallic mold are always higher than those in the ceramic-ceramic mold. Both the curves rapidly increase in the vicinity of the center of the sample $(y=10 \mathrm{~mm})$. It is noted that $V_{\mathrm{T}}$ is given by $V_{\mathrm{T}}=\dot{T} / G$ where $\dot{T}$ and $G$ are the cooling rate and the temperature gradient at $T_{\gamma}$ position, respectively. At the center position, $G$ becomes null due to symmetric heat flow toward both sides and $V_{\mathrm{T}}$ accordingly becomes infinitely large at $y=10 \mathrm{~mm}$ in these casting conditions. The solid line in Fig. 5(b) represents the result for the metallic-ceramic mold casting. The metallic mold and ceramic mold sides correspond to $y=0$ and $20 \mathrm{~mm}$, respectively. The behavior of $V_{\mathrm{T}}$ in Fig. 5(b) is asymmetric with respect to $y=10 \mathrm{~mm}$. In the vicinity of the ceramic mold, $V_{\mathrm{T}}$ takes high values and it rapidly increases as $T_{\gamma}$ position moves inward ( $-y$ direction), which are associated with low values of $G$ in this region due to slow heat transfer.

The arrows at the top part of Fig. 5 indicate the average transition depths in the $\gamma$ grain structure. The critical condition should be realized at these depths. From the comparison between the curve of $V_{\mathrm{T}}$ and the transition depth, the critical value of $V_{\mathrm{T}}$ was determined to be $V_{\mathrm{T}}=0.54 \mathrm{~mm} / \mathrm{s}$ in the metallic-metallic mold, $V_{\mathrm{T}}=0.37 \mathrm{~mm} / \mathrm{s}$ in the ceramic-ceramic mold and $V_{\mathrm{T}}=0.58 \mathrm{~mm} / \mathrm{s}$ in the metallic-ceramic mold casting.

In Eq. (1), $d_{\mathrm{FCG}}$ represents the short-axis diameter of the FCG. As already explained, $d_{\mathrm{FCG}}$ is almost equivalent to the distance between the neighboring dendrite trunks along the direction perpendicular to the heat flow. Therefore, $d_{\text {FCG }}$ can be estimated from the dendrite structure. The dashed lines in Fig. 5 shows the average distance between the dendrite trunks $\lambda$ measured from the microstructural observations. In Fig. 5(a), the values of $\lambda$ in the metallic-metallic mold casting are always lower than the values in the ceramic-ceramic mold casing, which can be explained by the higher cooling rate in the former case. The distribution of $\lambda$ in the metallic-ceramic mold casting is asymmetric as shown in Fig. 5(b). It is noted that $\lambda$ is essentially determined by the cooling condition during the $\delta$ solidification process. The cooling condition is not significantly asymmetric in the very beginning of the solidification and accordingly the distribution of $\lambda$ in Fig. 5(b) is not saliently asymmetric compared with the behavior of $V_{\mathrm{T}}$. This is consistent with the fact that the columnar dendrites develop from the ceramic mold as well as the metallic mold (Fig. 4(b)). The critical value of $d_{\mathrm{FCG}}$ at the transition depth was estimated to be $d_{\mathrm{FCG}}=140 \mu \mathrm{m}$ in the metallic-metallic mold, $160 \mu \mathrm{m}$ in the ceramic-ceramic mold and $200 \mu \mathrm{m}$ in the metallic-ceramic mold casting.

\subsection{Test of the critical condition}

The critical values of $V_{\mathrm{T}}$ and $d_{\mathrm{FCG}}$ were estimated for each casting condition as 
discussed above. The remaining unknown parameter in Eq. (1) is only $M\left(T_{\gamma}\right)$. Although the critical values of $V_{\mathrm{T}}$ and $d_{\mathrm{FCG}}$ depend on the casting condition as described above, $M\left(T_{\gamma}\right)$ should be a unique value regardless of the casting condition. It is noted that $M\left(T_{\gamma}\right)$ can now be estimated by substituting the critical values of $V_{\mathrm{T}}$ and $d_{\mathrm{FCG}}$ and $\xi=0.45$ into Eq. (1). Then, the critical condition given by Eq. (1) can be validated if $M\left(T_{\gamma}\right)$ estimated from Eq. (1) takes a unique value in all the casting conditions. We test the validity of Eq. (1) in this way. The estimated values of $M\left(T_{\gamma}\right)$ for three casting conditions are shown in Fig. 6 where the horizontal axis is the temperature and the values of $M\left(T_{\gamma}\right)$ are plotted at $T=T_{\gamma}$. The error bar is added for each plot by counting the different values of $V_{\mathrm{T}}$ and $d_{\text {FCG }}$ associated with the variation of the transition depth in each casting experiment. It is very important to note in Fig. 6 that the all the values of $M\left(T_{\gamma}\right)$ are almost identical within the range of the experimental error. That is, a unique value of $M\left(T_{\gamma}\right)$ is obtained even though the critical values of $V_{\mathrm{T}}$ and $d_{\mathrm{FCG}}$ are different depending on the casting condition. Therefore, the validity of the critical condition for the CCG formation given by Eq. (1) is successfully demonstrated by the present casting experiments.

The following point related to the quantitative accuracy of the critical condition is discussed. The temperature and concentration dependences of the kinetic constant $M(T)$ were investigated and the empirical equation of $M(T)$ was obtained in a recent work on the austenite grain growth [19]. The dashed line in Fig. 6 represents the values of $M(T)$ for the 0.2 mass\%C steel of our focus calculated from this empirical equation. The calculated results are extended to temperatures higher than the liquidus temperature of this steel, $T_{l}$. In Fig. 6, furthermore, $M(T)$ for pure iron [20] is plotted as additional reference data. It is noted that the values of $M\left(T_{\gamma}\right)$ estimated in the present study are fairly close to the calculated value for 0.2 mass\%C steel at $T=T_{\gamma}$. Although not shown here, when $\xi=0.78$ is utilized in Eq. (1) instead of the corrected value of $\xi=0.45$, our data in Fig. 6 take higher values, deviating from the empirical equation. Hence, it is understood by comparison between our data and the empirical equation that the present modification in fact improves the accuracy of Eq. (1). On the other hand, a care must be paid to the accuracy of the empirical equation. One cannot deny the possibility that the values of empirical equation have a margin of error. In the work on the austenite grain growth [19], the empirical equation for $M(T)$ was obtained not by investigating the time evolution of isothermal grain growth at several holding temperatures but by measuring the final grain size after cooling processes with an assumed initial grain size. In Fig. 6, our data are slightly higher than the empirical equation at $T=T_{\gamma}$. At this point, it is not clear whether or not this slight deviation falls within the experimental error. This point remains to be addressed in a future work in order to improve the quantitative accuracy 
of the critical condition for the CCG formation.

\section{Summary}

The formation of coarse columnar $\gamma$ grain (CCG) structure in peritectic solidified carbon steels is one of the serious problems in the field of casting of steels. There has been no guiding principle developed to avoid the formation of CCGs. Our recent works revealed that the CCGs form by the discontinuous grain growth from fine columnar $\gamma$ grains (FCGs) [7,8]. Furthermore, it was shown by the phase-field simulations that the CCG formation by the discontinuous growth takes place when $V_{\mathrm{T}} \leq$ $V_{\text {CCG }}$ is satisfied [9]. Here, $V_{\mathrm{T}}$ is the migration velocity of $T_{\gamma}$ position and $V_{\text {CCG }}$ is the migration velocity of the FCG/CCG region boundary (FCRB) and it is given by $V_{\mathrm{CCG}}=$ $M\left(T_{\gamma}\right) /\left(\xi d_{\mathrm{FCG}}\right)$ from the theory of the grain growth. The critical condition for the formation of CCGs was then put forward as given by Eq. (1). However, the effect of liquid phase on $V_{\mathrm{CCG}}$ was omitted in the previous numerical work [9] and, importantly, the experimental verification of Eq. (1) was lacking. In this study, the critical condition was reevaluated by taking the effect of the liquid phase into account, which yields the modification in the value of $\xi$ related to the shape of FCRB. Then, the validity of the modified critical condition was tested by means of three casting experiments with different molds and it was successfully substantiated.

It should be pointed out that as reported in our previous work [8], the formation of CCG does not take place by the mechanism of discontinuous grain growth in hyperperitectic carbon steels having high carbon concentrations, more specifically higher than 0.43 mass\% $\mathrm{C}$ in the cooling conditions similar to those in practical continuous casting. In such steels having high carbon concentrations, the formation of columnar $\gamma$ grains originates from the preferential growth of $\gamma$ dendrite instead of $\delta$ dendrite. As a result, the as-cast $\gamma$ grain structure is closely related to the size and shape of $\gamma$ dendrites [8]. In this case, Eq. (1) cannot be utilized. The present findings should be applied to peritectic solidified steels having lower carbon concentrations which are more prone to exhibiting the coarse columnar $\gamma$ grains due to the discontinuous grain growth. Hence, Eq. (1) is applicable to such peritectic solidified steels. We expect that based on Eq. (1) it should be possible to design the cooling condition and/or chemical compositions of steels so as to prevent the formation of CCGs in various casting processes including the continuous casting. However, how much adjustment of cooling condition is required for the prevention of CCG should be dependent on several factors such as steel compositions and casting conditions. A substantial change of cooling conditions should be unrealistic especially in continuous casting processes. Hence, if the 
prevention of CCG requires such a substantial change of cooling conditions, it should be reasonable to adjust the steel compositions since the steel compositions should affect all the variables in Eq. (1), viz., $V_{\mathrm{T}}, M\left(T_{\gamma}\right)$ and $d_{\mathrm{FCG}}$. In this regard, the dependences of three quantities, $V_{\mathrm{T}}, M\left(T_{\gamma}\right)$ and $d_{\mathrm{FCG}}$ on the cooling conditions and chemical compositions need to be clarified in detail. The investigations of these dependences should be one of the important tasks tackled in future works to make the important progress in controlling the as-cast $\gamma$ grain structure.

\section{Acknowledgements}

This work is partly supported by from The $19^{\text {th }}$ Steelmaking Committee's Research Grant, JSPS, Japan. 


\section{References}

[1] Reiter J, Bernhard C, Presslinger Mater Character 2008; 59: 737.

[2] Kobayashi Y, Iwasaki, S. Nakazato K, Hibaru T, Kuroda S, Sakuma N, Yoshida N, Nagai K. ISIJ Int 2008; 48: 344.

[3] Schmidt L, Josefsson Å. Scand J Metall 1974; 3: 193.

[4] Mintz B, Arrowsmith JM. Met Technol, 1979; 6: 24.

[5] Maehara Y. Yasumoto K, Sugitani Y, Gunji K. Trans ISIJ 1985;25: 1045.

[6] Crowther DN Mintz B. Mater Sci Tehcnol 1986; 2: 951.

[7] Tsuchiya S, Ohno M, Matsuura K, Isobe K. Acta Mater 2011; 59:3334.

[8] Tsuchiya S, Ohno M, Matsuura K. Acta Mater 2012; 60: 2927.

[9] Ohno M, Tsuchiya S, Matsuura S. Acta Mater 2011; 59: 5700.

[10] Humphreys FJ. Acta Mater 1997; 45: 4231.

[11] Hillert M. Acta Metall 1965; 13: 227.

[12] Hondros ED. Proc R. Soc London A 1965; A286: 479.

[13] Sun Y, Asta M. Hoyt JJ, Mendelev MI, Srolovitz DJ. Phys Rev B 2004; 69: 020102.

[14] Ohno M, Ohyama S, Matsuura K. in preparation.

[15] Ohno M, Matsuura S. ISIJ Int 2008; 48: 1373.

[16] Onaka I. Introduction to computer heat transfer and solidification analysis, Tokyo: Maruzen; 1985.

[17] CompuTherm LLC: http://www.computherm.com/. Last accessed date: 22/04/2013.

[18] The Japan Institute of Metals. Kinzoku Databook, $4^{\text {th }}$ ed. Tokyo: Maruzen; 2004.

[19] Bernhard C, Reiter J, Presslinger H. Metall Mater Trans B 2008; 39B: 885.

[20] Yoshida N, Kobayashi Y, Nagai K. Tetsu-to-Hagané 2004; 90: 198. 

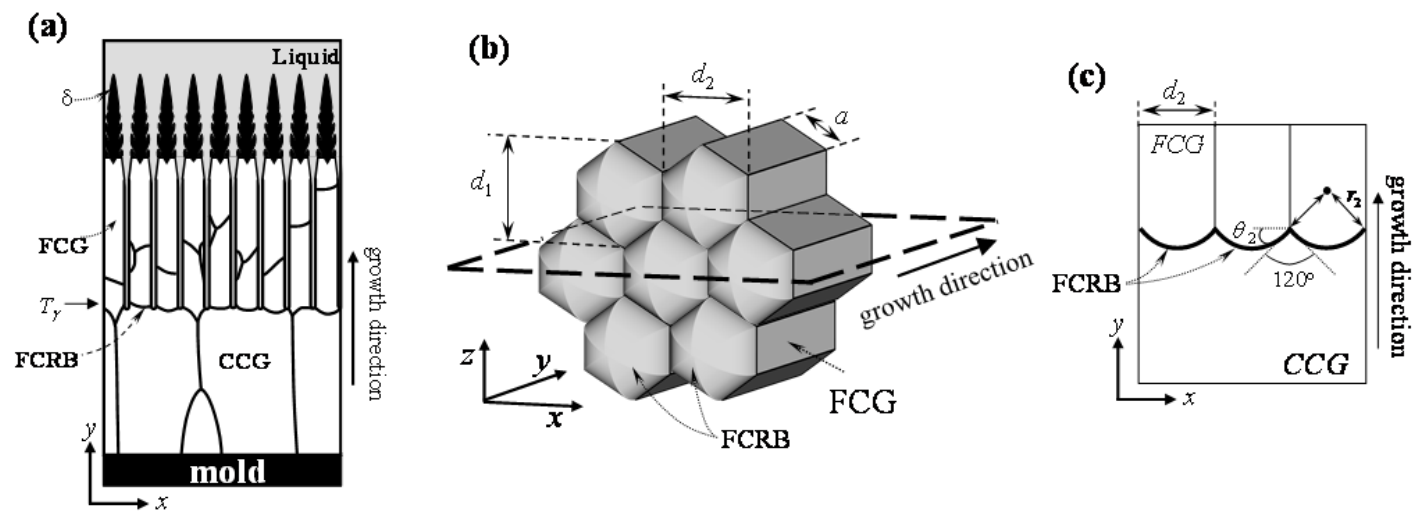

Fig. 1. (a) Schematic illustration of the CCG formation process by the discontinuous grain growth, (b) three-dimensional shape of FCRB and (c) two-dimensional shape of FCRB on $x-y$ plane without liquid. $\theta_{2}$ is an angle between $x$-axis and the FCRB near the triple junction. 
(a)

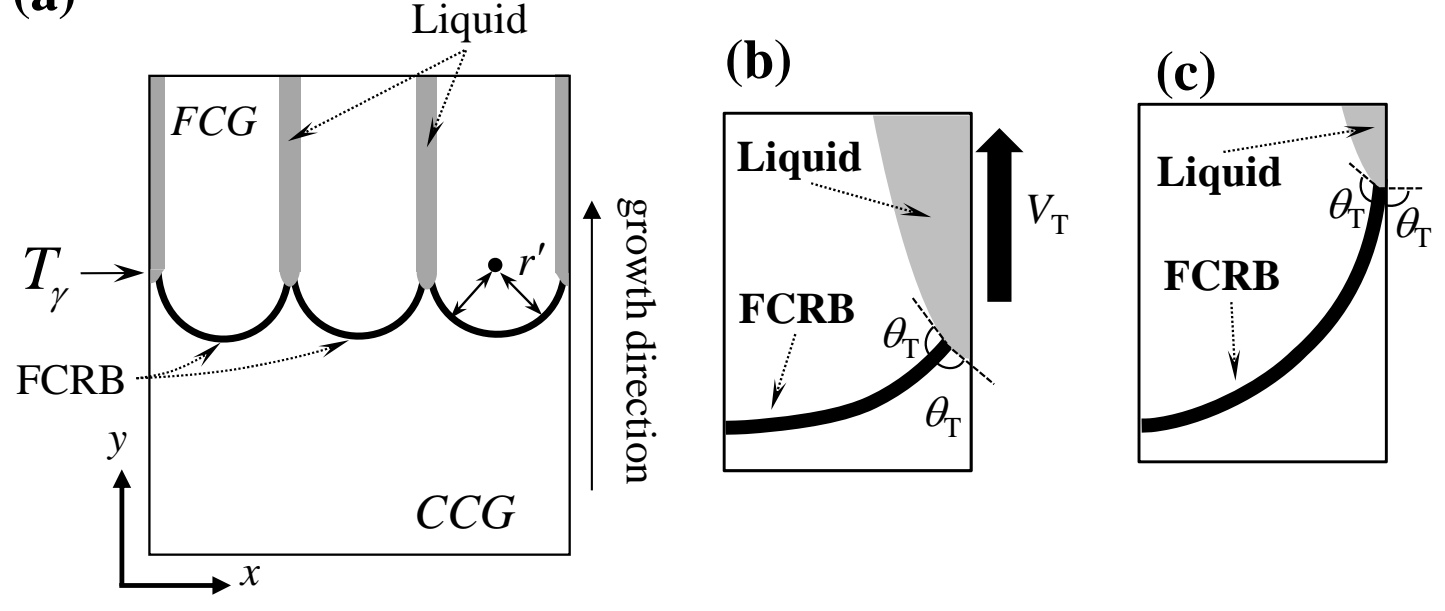

Fig. 2. (a) Two-dimensional shape of FCRB on $x-y$ plane with liquid phase. (b,c) local shapes of FCRB with the liquid at a time (b) $t=t_{1}$ and (c) $t=t_{2}\left(t_{1}<t_{2}\right)$. 
(a)
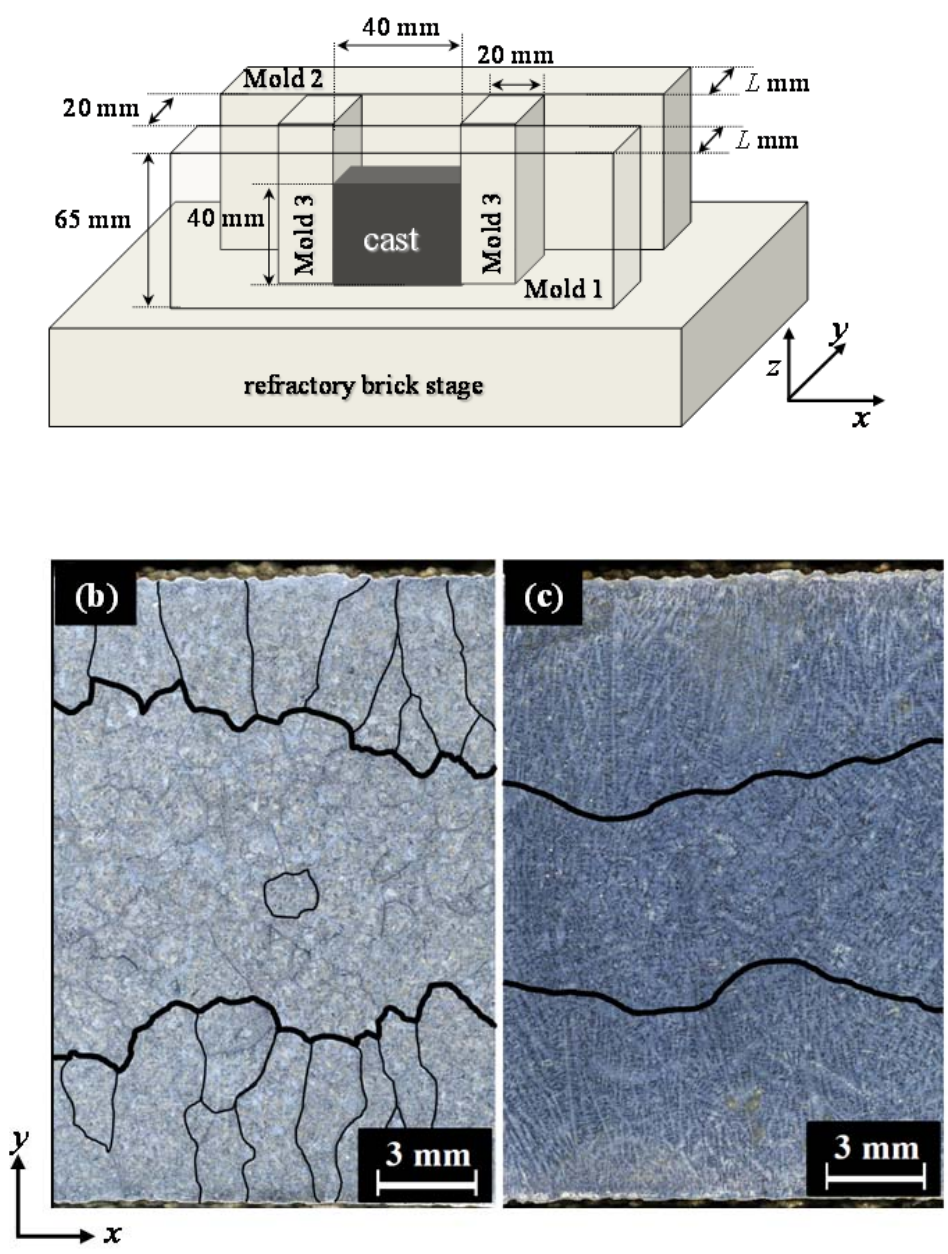

Fig. 3. (a) Schematic illustration of the casting molds employed in this study. (b) As-cast $\gamma$ grain structure and (c) dendrite structure at the same position in the sample of the ceramic-ceramic mold casting. Some grain boundaries are traced by thin lines for visual aid in Fig. (b). In Figs. (b) and (c), the thick lines represent the position where the columnar structure changes to the equiaxed structure. 

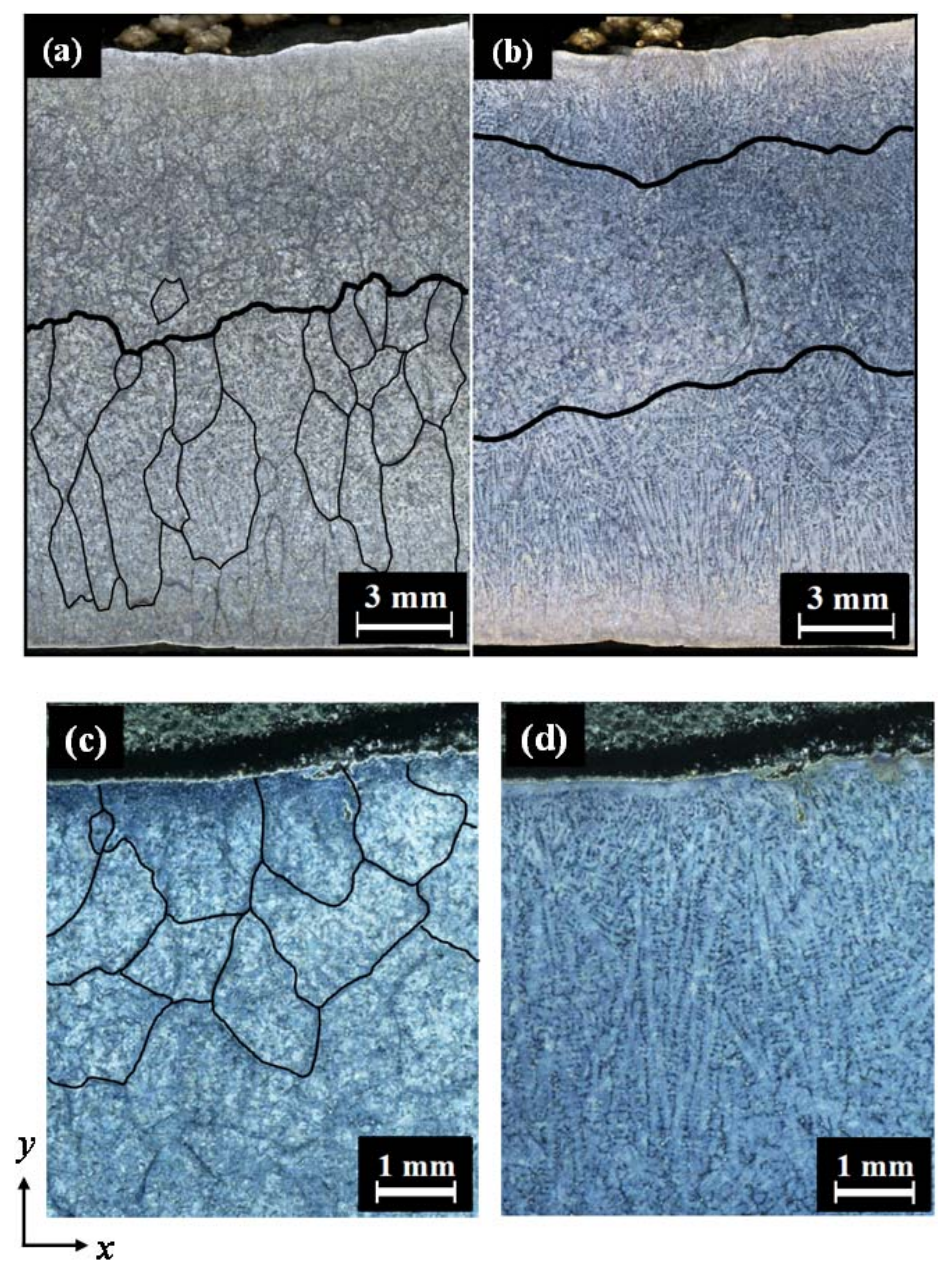

Fig. 4. (a) As-cast $\gamma$ grain structure and (b) dendrite structure at the same position in the sample of the metallic-ceramic mold casting. The bottom and top sides corresponds to the metallic and ceramic mold sides, respectively. Some grain boundaries are traced by thin lines for visual aid. In both figures, the thick lines represent the position where the columnar structure changes to the equiaxed structure. (c) and (d) Enlargements of as-cast $\gamma$ grain structure and dendrite structure, respectively, in the vicinity of the ceramic mold. 
(a)

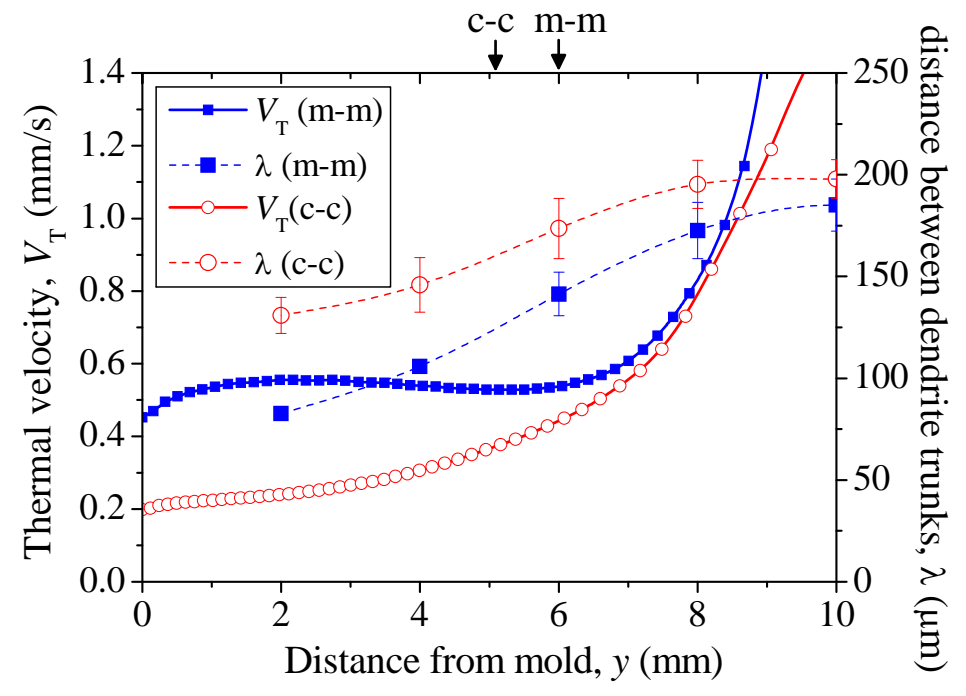

(b)

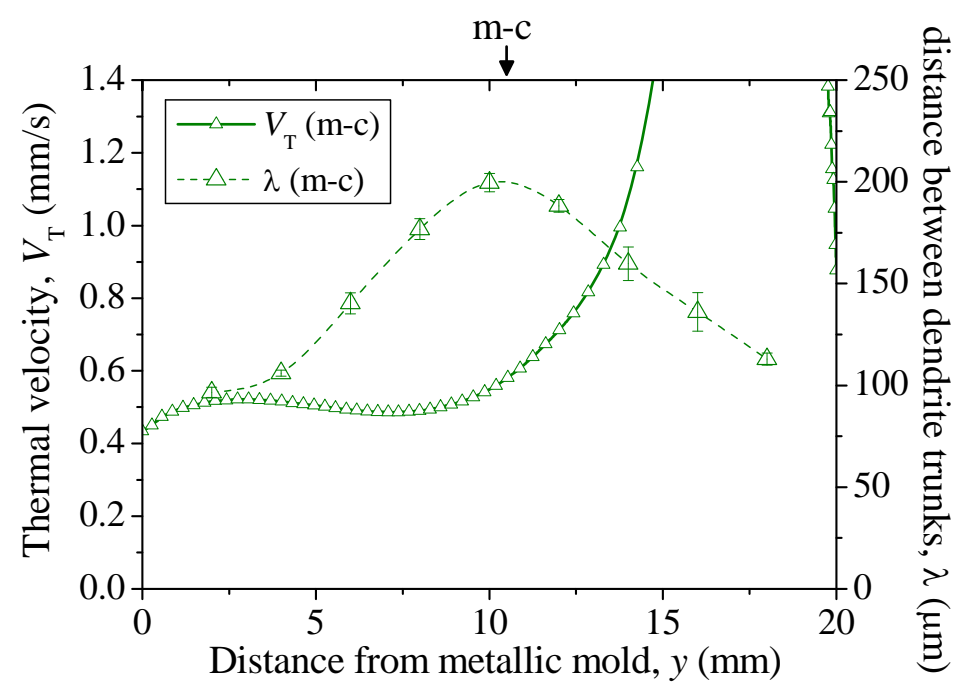

Fig. 5. Calculated thermal velocities (solid lines) and measured distance between the dendrite trunks (dashed lines) in (a) metallic-metallic mold (square plots), ceramic-ceramic mold (circle plots) and (b) metallic-ceramic mold (triangle plots) casting. The arrows on the top of each figure indicate the transition depth where the $\gamma$ grain structure changes from CCG to EG. In these figures, m-m, c-c and m-c denote the metallic-metallic, ceramic-ceramic and metallic-ceramic casting experiments. 


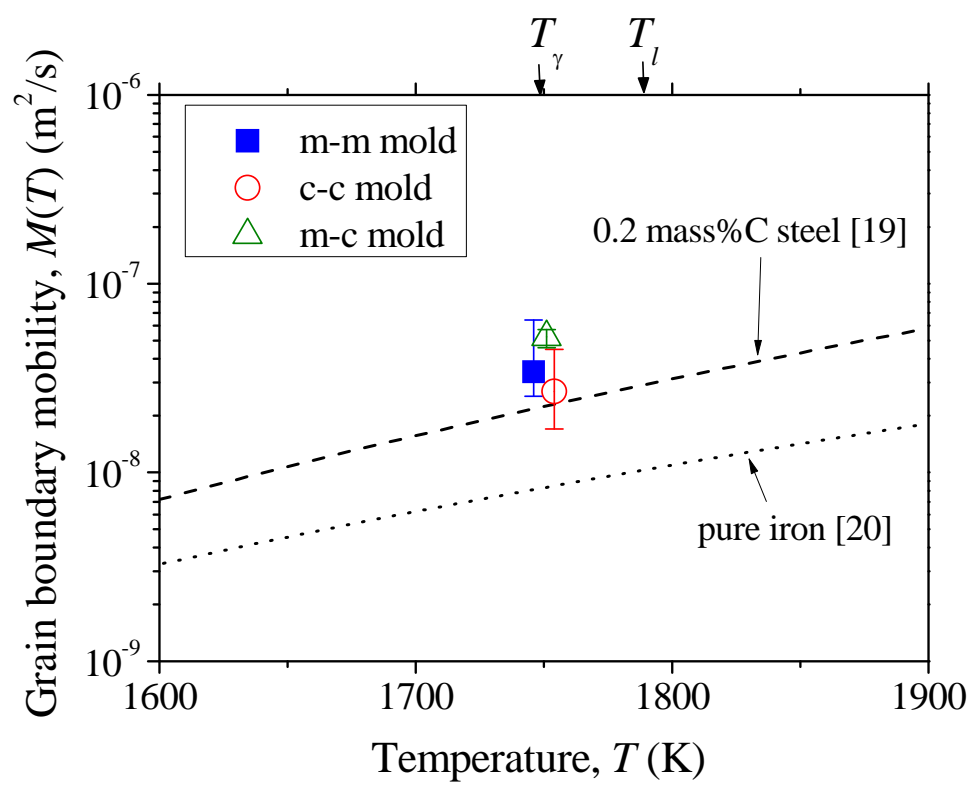

Fig. 6. Kinetic constant for the grain growth at $T_{\gamma}, M\left(T_{\gamma}\right)$, estimated from Eq. (1) (plots) and the temperature dependence of $M(T)$ for 0.2 mass\% C steel (dashed line) [19] and pure iron (dotted line) [20]. The liquidus temperature, $T_{l}$, and $T_{\gamma}$ of 0.2 mass\%C steel are indicated at the top of figure by the arrows. 